\title{
Review of Metamodeling Techniques for Product Design with Computation- intensive Processes
}

\author{
G. Gary Wang, S. Shan \\ Dept. of Mechanical and Manufacturing Engineering \\ The University of Manitoba \\ Winnipeg, MB, R3T 5V6 \\ Tel: (204) 474-9463 Fax: (204) 275-7507 \\ Email: gary_wang@umanitoba.ca
}

\begin{abstract}
Computation-intensive design problems are becoming increasingly common, especially for large-size manufacturers such as those in the aerospace, automotive, and electronics industries. The computation burden is often caused by expensive analysis and simulation processes. Approximation or metamodeling techniques are often used to model these computationintensive processes in order to improve efficiency. This work will review the current state-of-the-art on metamodeling-based techniques in support of product design. The review is organized from a practitioner's perspective according to the role of metamodeling in supporting design. Challenges and future development of metamodeling will also be analyzed and discussed.
\end{abstract}

\section{Introduction}

To address the global competition, manufacturing companies strive to produce better and cheaper products more quickly. For complex systems such as aircraft, the design is intrinsically a daunting optimization task often involving multiple disciplines, multiple objectives, computation-intensive processes for product simulations, and both discrete and continuous design variables. Just taking the computation challenge as an example, it is reported that it takes Ford Motor Company about 36-160 hrs to run one crash simulation (Gu 2001). For a twovariable optimization problem, assuming in average 50 iterations are needed by optimization and assuming each iteration needs one crash simulation, the total computation time would be 75 days to 11 months, which is unacceptable in real practice. Despite continual advances in computing power, the complexity of analysis codes, such as finite element analysis (FEA) and computational fluid dynamics (CFD), seems to keep pace with computing advances (Koch et al. 1999). In the past two decades, USA invested significant amount in the research on design optimization through NASA, Department of Defense (DOD), and National Science Foundation (NSF) to overcome mainly the computation barrier.
In recent years, the approximation-based optimization method has attracted many attentions. This approach approximates computation-intensive functions with simple models. With a simple model, often called metamodel, classic optimization methods can then be effectively applied to search for the optimum. Such a method is therefore referred as metamodeling-based optimization method. The advantages of this approach are manifold: 1) the efficiency of optimization is greatly improved with metamodels; 2) because the approximation is based on sample points, which could be obtained independently, parallel computation is supported (Assuming an optimization requires 50 expensive function evaluations and each takes 2 hours, these 50 evaluations can be computed in parallel and thus the total amount of time is 2 hours as compared to 100 hours.); 3) the approximation process can help study the sensitivity of design variables, and thus give engineers insights to the problem; and 4) this method can handle both continuous and discrete variables.

Continuing on an earlier review (Barthelemy and Haftka 1993), Haftka and coauthors (Haftka et al. 1998) discussed in depth the relation between experiments and optimization, i.e., the use of optimization to design experiments, and the use of experiments to support optimization. It also dedicated a chapter talking about metamodeling-based design optimization (MBDO), though slightly different terminologies were used in the paper. The benefits of MBDO were elaborated as follows: 1) it is easier to connect proprietary and often expensive simulation problems; 2) parallel computation becomes simple as it involves running the same simulation at many deign points; 3 ) building metamodels can better filter numerical noises than derivative-based methods; 4) the metamodel renders a view of entire design space; and 5) it is easier to detect errors in simulation as the entire design domain is analyzed. Simpson et al. (Simpson et al. 2001c) gave a very focused review on metamodels and MBDO by going through many popular sampling methods (or experimental design methods), approximation models 
(metamodels), metamodeling strategies, and applications. Guidelines and recommendations were also given at the end of the paper. A panel discussion about the topic was held in 2002 in the 9th AIAA/USAF/NASA/ISSMO Symposium on Multidisciplinary Analysis \& Optimization in Atlanta. The summary of the panel discussion was archived in (Simpson et al. 2004). Four future research directions were elaborated as 1) sampling methods for computer experiments, 2)visualization of experimental results, 3) capturing uncertainty with approximation methods, and 4) high dimensional problems.

In the past few years, new developments in MBDO have been continuously coming forth. It also seems also as more and more of MBDO approaches being developed, the gap between the research community and design engineers keeps widening. This work intends to provide a review of recent development in this area to cater the needs of design engineers. Therefore, the review will be organized in a way to provide a reference of MBDO for a practitioner.

\section{Roles of MBDO In Support of Product Design}

Intensive research has been done in employing metamodeling techniques to search for design optimum. These include research on sampling, metamodels, model construction techniques, model validation, iterative sampling, optimization methods in support of different types of optimization problems, design visualization, and so on. Through the years it becomes clear that MBDO is in a decision-support role for design engineers. What are the supporting functions that MBDO can provide? From authors' experience and informal interview with design engineers, with reference to literatures, the following lists some of the areas that MBDO can play a role. Such a list is not meant to be exclusive or exhaustive. New areas are believed to emerge as the industry gradually adopts MBDO.

- Approximation of computation-intensive processes to reduce computation costs

- Enhancement of the understanding of the design problem including visualization and sensitivity study

- Assistance in better defining the design optimization model through variable and space reduction

- Support of various optimization needs, e.g., global optimization, multi-objective optimization, multidisciplinary design optimization, probabilistic optimization, etc.

For each of the above-mentioned areas, related recent development in metamodeling and MBDO will be reviewed. General consensus has been reached thus far in the research community will be given. New trends and directions in each area will also be discussed.

\section{Approximation of Computation-Intensive Processes}

Approximation, or metamodeling, is the key to metamodeling-based design optimization. Conventionally the goal of approximation is to achieve a metamodel as accurate as possible at a reasonable cost. With new developments, such a goal shifts if the users' ultimate objective is to obtain the optimal design rather than the entire model. In this section, we assume that the goal remains to obtain an accurate approximation model of a computation-intensive function, and leave the optimization to other sections.

With the aim to reduce the computation cost for computation-intensive design problems, current metamodeling related research is roughly along three directions. The first direction is on the identification and development of a group of design alternatives for approximation, or experimental designs The second aims at developing a "better" metamodel for different applications. The third takes a dynamic or iterative approach in which sampling and metamodeling are alternated in order to achieve simultaneously the overall efficiency and accuracy.

\section{Sampling}

Due to the many differences between computer experiments and physical experiments as discussed in (Simpson et al. 2004), this work simply uses the term "sampling" to refer to experimental design. Among various groups of developed experimental designs, the Central Composite Design (CCD) has been widely used. However, the number of points in CCD increases exponentially with the number of design variables, which proves to be inefficient for high-dimensional design problems. Though there are small composite designs (SCD) that consist of a faction of CCD points, the SCD has significant difficulty in estimating linear and interaction coefficients (Chen 1995; Myers and Montgomery 1995). Chen (Chen 1995) designed a nonstandard CCD method and positive results have been achieved. Besides CCD, alphabetical optimal designs, especially D-optimal designs, are also widely used (Giunta et al. 1997; Johnson et al. 1990; Mitchell 1974). Myers and Montgomery (Myers and Montgomery 1995) identified the pitfalls of the D-optimality designs, which have only model-dependent D-efficiency and do not address prediction variance. Moreover, for second-order models, the D-criterion often does not allow any (or many) center runs. This often leaves large variance in the design center. As computer experiments involve mostly systematic error rather than random errors as in physical experiments, Sacks et al. (Sacks et al. 1989b) stated that in the presence of systematic rather than random error, a good experimental design tends to fill the design space rather than to concentrate on the boundary. They also stated that standard designs, e.g. CCD and Doptimality designs, can be inefficient or even 
inappropriate for deterministic computer codes. Simpson et al. (Simpson et al. 2001c) confirmed that a consensus among researchers is that experimental designs for deterministic computer analyses should be space filling.

There are four groups of space filling methods being used in the literature, including orthogonal arrays (Hedayat et al. 1999; Owen 1992; Taguchi et al. 1993), various Latin Hypercube Designs (Iman and Conover 1980; McKay et al. 1979; Park 1994; Tang 1993; Ye et al. 2000), Hammersley sequences (Kalagnanam and Diwekar 1997; Meckesheimer et al. 2002), and uniform designs (Fang et al. 2000). Code for generating orthogonal arrays is available online at http://lib.stat.cmu.edu/design/owen.html. Hammersley sampling is found to provide better uniformity than Latin hypercubes. Several uniform designs are available online at URL: http://www.math.hkbu.edu.hk/UnifromDesign. $\quad$ A comparison of these sampling methods is in Ref. (Simpson et al. 2001a).

The Monte Carlo Simulation (MCS) method, which is a random sampling method, is still a popular sampling method in MBDO in industry, regardless of its inefficiency. It is probably because of the fact that no one is sure about the adequate sample size at the outset of MBDO for any black-box function. Improved from the Monte Carlo simulation method, the importance sampling (IS) bears the potential of improving its efficiency while maintain the same level of accuracy of MCS (Au and Beck 1999). Zou and colleagues developed an indicator response surface based method, in which IS is performed in a reduced region around the limit state (Zou et al. 2002; Zou et al. 2003). The authors recently developed an innovative sampling method which achieves impressive efficiency and accuracy for global optimization, multi-objective optimization, and reliability assessment (Shan and Wang 2004; Wang et al. 2005; Wang et al. 2004). With its original inspiration from (Fu and Wang 2002), this sampling method is space filling and reflects the goal of sampling; it is a more aggressive MCS method.

\section{Metamodeling}

Metamodeling evolves from classical Design of Experiments (DOE) theory, in which polynomial functions are used as response surfaces, or metamodels. Besides the commonly used polynomial functions, Sacks et al. (Sacks et al. 1989a; Sacks et al. 1989b) proposed the use of a stochastic model, called Kriging (Cresssie 1988), to treat the deterministic computer response as a realization of a random function with respect to the actual system response. Neural networks have also been applied in generating the response surfaces for system approximation (Papadrakakis et al. 1998). Other types of models include Rational Basis Functions (RBF) (Dyn et al. 1986), Multivariate Adaptive Regression Splines
(MARS) (Friedman 1991), and inductive learning (Langley and Simon 1995). A combination of polynomial functions and artificial neural networks has also been archived in (Varadarajan et al. 2000). So far there is no conclusion about which model is definitely superior to the others. However, much more insight on various models has been gained through a number of studies (Giunta and Watson 1998; Jin et al. 2001; Koch et al. 1999; Simpson et al. 2001b; Simpson et al. 2001c). Among various models, Kriging and second-order polynomials are the most intensively studied. The general consensus is that the Kriging model is more accurate for nonlinear problems but difficult to obtain and use. On the contrary, the polynomial model is easy to construct, clear on parameter sensitivity, and cheap to work with but is less accurate than the Kriging model (Jin et al. 2001; Simpson et al. 2001c). There are also metamodels on fuzzy numbers (Huber et al. 1996; Madu 1995).

Recently, a new model called Support Vector Regression (SVR) was used and tested (Clarke et al. 2005). SVR achieved high accuracy over all other metamodeling techniques including kriging, polynomial, MARS, and RBF over a large number of test problems. It is not clear, however, what are the fundamental reasons that SVR wins out. Messac and his team developed an extended RBF model (Mullur and Messac 2005) by adding extra terms to a regular RBF model to increase its flexibility. It is claimed that this extended RBF applies to almost all problems. Also recently, Tuner and Crawford proposed NURBS metamodels as a generic metamodel (Turner and Crawford 2005). The interests on Kriging remain high with more in-depth studies (Kleijnen and van Beers 2003; Martin and Simpson 2005). A well written Kriging modeling code (in Matlab) was downloadable from the internet URL: http://www2.imm.dtu.dk/ hbn/dace/ (Lophaven et al. 2002).

If gradient information can be reliably and inexpensively obtained, gradient information can be utilized in metamodeling (Koehler 1997; Morris et al. 1993). A multipoint approximation (MPA) strategy also received some attention (Rasmussen 1998; Shin and Grandhi 2001; Toropov and Filatov 1993; Wang et al. 1996). MPA uses blending function to combine multiple local approximations, and usually gradient information is also used in metamodeling.

From the current studies, it seems that a flexible and generic metamodeling approach is sought after. Such a metamodeling approach can be further extended to include metamodels of mixed discrete and continuous variables, metamodel of variable fidelity across the entire or sub-domains of design spaces (Leary et al. 2003), tuneable metamodels (more will be discussed later), and so on. 


\section{Iterative Metamodeling}

From the practice perspective, no matter which metamodel is used, there is always an accuracy concern from the sample size and distribution, and the difference between the metamodel and the real function. Therefore, methods that can iteratively improve the accuracy of the metamodel have been under development (Dennis and Torczon 1996). Regardless of which metamodeling technique is used for a specific problem, it is observed that the modeling efficiency and accuracy are directly related to the design space (i.e., the approximation space for metamodels). A branch of research under the metamodeling umbrella thus aims at developing methods that can gradually reduce the design space to improve the modeling accuracy. Two types of design space reduction schemes are seen in the literature. One is to reduce the dimensionality of the design space by reducing the number of design variables. The early work of Box and Draper (Box and Draper 1969) introduces a method to gradually refine the response surface to better capture the real function by "screening" out unimportant variables. Welch et al. (Welch et al. 1992) documents a systematic approach for screening the variables. The variablecomplexity response surface modeling method uses analyses of varying fidelity to reduce the design space to the region of interest (Balabanov et al. 1999; Giunta et al. 1997). The reduction of dimensionality is very important as it aims to break the "curse of dimensionality"; however, dimensionality is difficult to reduce especially for multidisciplinary design problems (Koch et al. 1999).

The other type of design space reduction seeks to reduce the size of the design space while assuming the dimensionality cannot be further reduced. Since the combined range of each design variable dictates the size of the design space, the larger the range for each design variable, the larger the design space; the larger the design space, the more difficult and costly to construct accurate metamodels. Engineers tend to give very conservative lower and upper bounds for design variables at the initial stage of setting up a design optimization problem. This is often due to the lack of sufficient knowledge of function behaviour and interactions between objective and constraint functions at the early stage of problem definition. Chen and her co-authors (Chen et al. 1997) develop heuristics to lead the surface refinement to a smaller design space. Wujek and Renaud (Wujek and Renaud 1998a; Wujek and Renaud 1998b) compare a number of move-limit strategies that all focus on controlling the function approximation in a more "meaningful" design space. Many researchers advocate the use of a sequential metamodeling approach using move limits (Toropov et al. 1996) or trust regions (Alexandrov et al. 1998; Rodríguez et al. 1998). For instance, the Concurrent SubSpace Optimization procedure uses data generated during concurrent subspace optimization to develop response surface approximations of the design space, which form the basis of the subspace coordination procedure (Renaud and Gabriele 1994). More recently, Pérez et al. (Pérez et al. 2002) transforms the matrix of second-order terms of a quadratic polynomial model into the canonical form. Samples are generated iteratively to update the approximation to maintain the model accuracy. Osio and Amon (Osio and Amon 1996) develop a multi-stage kriging strategy to sequentially update and improve the accuracy of surrogate approximations as additional sample points are obtained. Mathematically rigorous techniques and trust regions are also being combined to develop several other metamodel management frameworks to manage the use of approximation models in optimization (Booker et al. 1999; Rodríguez et al. 2001). Finally, Schonlau, et al. (Schonlau et al. 1998) describe a sequential algorithm to balance local and global searches using approximations during constrained optimization. Sasena et al. (Sasena et al. 2002) uses Kriging models for disconnected feasible regions. Knowledge is also incorporated in the identification of attractive design space (Gelsey et al. 1998).

In recent years, the lead author and his colleagues have developed the Adaptive Response Surface Method (ARSM), which systematically reduces the size of the design space by discarding portions of it that correspond to objective function values larger than a given threshold value at each modeling-optimization iteration (Wang 2003; Wang et al. 2001). Thus, the design space is gradually reduced to the neighbourhood of the global design optimum. The design space reduction procedure is characterized by its systematic consideration of the interrelationship between design variables. The improved ARSM applies inherited Latin Hypercube Designs (LHD) for sampling, which greatly reduced the number of samples required (Wang 2003). A heuristic hierarchical metamodeling approached was also developed to take advantage of both polynomial and kriging models (Wang and Simpson 2004).

To improve the efficiency of Kriging modeling, Shin and Diwekar uses re-weighting to update a kernel density estimator when new sample points are obtained. The metamodeling process needs not to be repeated and thus the efficiency of metamodeling is improved (Sahin and Diwekar 2004). Often recalibrated composite approximation models are also used in support of optimization (Ellman et al. 1997)

\section{Model Validation and Assessment}

Model validation and assessment has been an interesting and yet challenging tasks for the metamodeling research community. Normally there are two only types of validation methods. One is to use additional sample points to check the predicted function value with the real ones. The other is so-called the cross-validation method, including leave-one-out or leave- $k$-out approaches. A recent work with a review on this topic is in Ref. 
(Meckesheimer et al. 2002). The validation of metamodels may share common challenges with the verification and validation of other computational models (Oberkampf and Trucano 2000; Roache 1998).

\section{Enhancement of the Understanding of the Design Problem}

Most today's design tools such as CAD aim at improve the productivity of a design engineer. The abstract relationship between design variables and product performances is only reflected in complex equations or models. Engineers usually only have a vague idea about such relationship and such a vague idea relies on experience. A common method an engineer will use to understand a design problem is through sensitivity analysis and "what if" questions. The sensitivity analysis, however, is based on a fixed condition with the varying of one variable. If the condition is changed, the sensitivity information will change as well. An engineer still cannot have an idea of the overall structure of the problem. The metamodeling approach can assist the engineer to gain insight to the design problem, currently, through two channels. The first is through the metamodel itself. Given the metamodel, one can analyze the properties of the metamodel to gain a better understanding of the problem. A good example is for the quadratic polynomial metamodel, if all the design variables are normalized to $[-1,1]$, magnitude of the coefficients in the metamodel indicates the sensitivity or importance of the corresponding term (Montgomery 1991). This is in fact used for screening. The second way of enhancing the understanding is through visualization.

Visualization of multi-dimensional data alone has been an interesting topic and many methods have been developed over the years (Keim and Kriegel 1996; Wong and Bergeron 1997). The visualization of design space with the assistance of metamodels, however, is recent. State University of New York (SUNY) at Buffalo developed a visual design steering method based on the concept of Graph Morphing (Winer and Bloebaum 2002a; Winer and Bloebaum 2002b). Eddy and Kemper, also from SUNY Buffalo, proposed cloud visualization for the same purpose (Eddy and Lewis 2002b). Also, SGI and Ford integrated parallel computation and metamodeling for rapid visualization of design alternatives. Penn State University's Applied Research Lab collaborated with JPL and Lockheed Martin Space Systems to develop visualization methods for multidimensional data sets and identifying Pareto Frontiers for multiobjective optimization problems (Simpson 2004; Stump et al. 2002). Other studies on visualization of multiobjective optimization results include Refs. (Eddy and Lewis 2002a; Mattson and Messac 2002).
The research on visualization needs to go more in-depth on understanding the needs of engineers and on designing the best intuitive interface in support of product design. Questions need to be answer include, to list a few: what are the visualization techniques are more intuitive and easily to understand? what data in design need to be visualized, why? what are the interactive means that the tool should and can provide to users? how will the visual aid help designer enhance the understanding the problem or better direct the design?

\section{Assistance in Defining Design Optimization Model}

Building a design optimization model is the first and yet critical step for design optimization. The quality of the optimization model directly affects the feasibility, cost, and effectiveness of the optimization process. It is apparent that metamodeling helps to provide an often explicit model of either the objective or constraint function to ease the optimization. Through the enhanced understanding of the design problem, as discussed in the last section, some of the objectives or constraints can be eliminated, combined, or modified. Moreover, metamodeling helps significantly in reducing the number of design variables and their range of search. This relates to the reduction of dimensionality and the size of design space, which have been addressed in the sub-section Iterative Metamodeling.

\section{Support of Various Optimization Needs}

Due to various needs in design, a design optimization problem could be global optimization in which the global optimum is sought after, multiobjective optimization in order to satisfy multiple design objectives, multidisciplinary design optimization where coupling between functions is present, or probabilistic optimization when uncertainties of variables are considered. In all these various optimization problems, metamodeling has been intensively used.

\section{Global Optimization}

A few methods have been developed for applying metamodeling in global optimization. One successful development is in Refs. (Jones et al. 1998; Schonlau et al. 1998), where the authors apply Bayesian method to estimate a kriging model, and then gradually identifies points in the space to update the model and perform the optimization. Their method, however, has to pre-assume a continuous objective function and a correlation structure among sample points. A Voronoi diagram based metamodeling method was proposed in which the approximation is gradually refined in ever smaller Voronoi regions and global optimum can be obtained (Hirokawa et al. 2002). Since Voronoi diagram is from computational geometry, the extension of this idea to problems with more than 3 variables may not be 
efficient. Global optimization based on multipoint approximation and intervals is performed in Ref. (Shin and Grandhi 2001). Metamodeling was also used to improve the efficiency of genetic algorithms (Hacker et al. 2001; Ong et al. 2003).

The authors and colleagues in the Concurrent Design Optimization Laboratory (CDOL) at the University of Manitoba developed an adaptive response surface method (ARSM) for global optimization (Wang 2003; Wang et al. 2001). ARSM systematically reduces the design space based on the metamodel until the metamodel is sufficiently accurate in a small region. Later, two methods from the area of artificial intelligence (AI) was integrated with metamodeling for the purpose of global optimization. First, a fuzzy clustering based method was developed (Wang and Simpson 2004), in which the design space was first reduced according to a polynomial metamodel and then a kriging model was refined in the reduced space until the global optimum is obtained. Second, a new tool, Rough Set, was employed to divide a design space to a number of attractive regions according to the design objective. Optima can be obtained in each of these regions (Shan and Wang 2003). A latest development from this group deviates dramatically from the general practice of using metamodeling in optimization, i.e., conventional optimization algorithms are applied to a metamodel. A novel, so-called Mode-Pursuing Sampling (MPS) method was developed (Wang et al. 2004), in which no existing optimization algorithm is applied. The optimization is realized through an iterative discriminative sampling process. Metamodels are only used as a guide for sampling. The demand for the accuracy of metamodels is significantly decreased.

\section{Multiobjective Optimization (MOO)}

Recent approaches to solve MOO problems with blackbox functions are to approximate each single objective function or directly approximate the Pareto optimal frontier (Li et al. 1998; Tappeta and Rosenberger 2001; Wilson et al. 2000; Yang et al. 2003). The accuracy of the Pareto optimal frontier depends on the accuracy of approximation models. Wilson et al. (Wilson et al. 2000) used the surrogate approximation in lieu of the computationally expensive analyses to explore the multiobjective design space and identify Pareto optimal points, or the Pareto set, from the surrogate. Li et al. (Li et al. 1998) used a hyper-ellipse surrogate to approximate the Pareto optimal frontier for bi-criteria convex optimization problems. If the approximation is not sufficiently accurate, then the Pareto optimal frontier obtained using the surrogate approximation will not be a good approximation of the actual Pareto optimal frontier. A latest work by Yang et al. (Yang et al. 2003) proposed the first framework managing approximation models in MOO. In the framework, a GA based method is employed with a sequentially updated approximation model. It differs from (Wilson et al. 2000) by updating the approximation model in the optimization process. The fidelity of the identified frontier solutions, however, is still built upon the accuracy of the approximation model. The work in Ref. (Yang et al. 2003) also suffers from the problems of the GA-based MOO algorithm, i.e., the algorithm has difficulty in finding frontier points near the extreme points (the minimum obtained by considering only one objective function). The author's group developed a sampling-based MOO method in which metamodels are used only as guidance (Shan and Wang 2004). This method rapid converges to the Pareto frontier. The fundamental difference of this approach from other work was elaborated in Ref. (Shan and Wang 2004)

\section{Probabilistic Design Optimization}

Probabilistic design optimization consists of both reliability-based design optimization (RBDO) and robust design optimization (RDO). Either one of them is an intensive research area. The use of metamodeling in these areas are wide. Instead of providing a detailed review of these areas, this work only summarizes some common themes with references to a few representative articles.

Reliability assessment is the building block for RBDO. Metamodels are often used to approximate expensive constraint functions, or the limit state function. Some recent work includes using importance sampling (IS) together with metamodels (Kloess et al. 2003; Zou et al. 2002). Zou and colleagues developed an indicator response surface based method, in which Monte Carlo Simulation is only performed in a reduced region around the limit state (Zou et al. 2003). The authors recently developed a more flexible discriminative sampling method with high efficiency and accuracy (Wang et al. 2005).

Metamodels are commonly used as a surrogate of expensive processes and probabilistic optimization processes are applied directly on the metamodels. Chen (Chen 1995) applied metamodeling in robust design in her $\mathrm{Ph} . \mathrm{D}$. dissertation and from then on developed a series of methods in the field (Chen et al. 1996; Chen et al. 2000; Varadarajan et al. 2000; Zhang et al. 2000). Booker continued on the surrogate management framework (SMF) (Booker et al. 1999) and applied it to reliability-based design (Booker et al. 2004). These methods are implemented into Boeing's Design Explorer tool. Choi and his group has been very active in this area (Youn and Choi 2004). They started to look into using metamodels in support of RBDO and RDO.

Recently, Jin et al. performed a study on using metamodeling techniques for optimization when uncertainties are present (Jin et al. 2003). It is found that a metamodel that is acceptable for deterministic optimization may not be acceptable for modeling the 
performance variations and the probability of constraint feasibility.

\section{Multidisciplinary Design Optimization (MDO)}

MDO has been a very crowded area, partially due to its broad definition. In real practice, MDO often involves a large number of design variables, computationallyintensive function evaluations, and coupling between disciplinary functions. All these features of MDO make metamodeling an attractive tool to be included in MDO methodologies (Giunta et al. 1997; Haftka et al. 1998; Sobieszczanski-Sobieski and Haftka 1997). A detailed survey of MDO in aerospace was given in Ref. (Sobieszczanski-Sobieski and Haftka 1997). Golovidov et al. discussed in detail the strategies of using metamodeling in MDO via a commercial software tool iSight (Golovidov et al. 1998). Batill et al. uses metamodels in solving the coordination between design subspaces (Batill et al. 1999). The use of metamodels in a current popular MDO methodology, Collaborative Optimization (CO), is archived in Ref. (Sobieski and Kroo 2000). The author's group used metamodeling to guide sampling design solutions that satisfy the coupling requirements between disciplines (Wang 2005). A metamodeling-based approach was also developed to search for boundaries of coupled state parameters. With such a boundary, the coupled MDO can be reformulated with as an uncoupled problem and design engineers can have an enhanced understanding of the problem.

\section{Challenges and Future Development}

\section{Large-scale Problems}

It is widely recognized that when the number of design variables is large, the total computation expense for metamodeling-based approaches makes the approaches less attractive or even infeasible (Koch et al. 1999). As an example, if the traditional central composite design (CCD) and a second-order polynomial function are used for metamodeling, the minimum number of sample points is $(n+1)(n+2) / 2$, which equals to the total number of coefficients of the quadratic model. Therefore, the total number of sample points required increases exponentially with the number of design variables. What are the characteristics of a large-scale problem? Are there special models and sampling schemes that best suit for large-scale problems (Srivastava et al. 2004)? Is decomposition the only method to crack down the largescale problem? What is the best decomposition strategy then? Is decomposition always feasible? It is in the authors' opinion that the limitation for large-scale problem is the most prominent problem in metamodeling-based design. New metamodeling techniques for large-scale problems, or a simple yet robust strategy to decompose a large-scale problem is needed.

\section{Metamodeling for Function Properties}

Currently metamodeling is only used for approximating the design variables and their performances, which are often used as an output of the "black-box" functions. It would be beneficial to have a model of gradient of the performance function, a model of curvatures, and so on. In the case of uncertainties, if might be helpful to have a metamodel of standard deviation to help probabilistic design optimization (Jin et al. 2003). Moreover, it would be even better if such a metamodel of certain function property can be derived from the metamodel of the performance function. Therefore, new innovative metamodel forms may be invented for this purpose.

\section{Tunable Metamodels}

Tunable metamodels refer to the case that approximation models can be "tuned" instead of "remodeled," when new sample points are added or the "black-box" function altered. It is found through practice that when new sample points are added, the metamodel has to be reconstructed as a brand new model. In kriging modeling, the computation cost for constructing a model is relatively high since it calls an optimization process to search for parameter estimations. If a model only needs fine tuning without reconstruction, the stability and efficiency of metamodeling can be greatly improved (Sahin and Diwekar 2004). Another situation relates to variable-fidelity "black-box" functions. If a metamodel is proved to be accurate for a low fidelity model, can it be tuned for a higher fidelity model? In the field of electrical engineering, a method called space mapping (Bakr et al. 2000) was developed, which builds a connection between low and high fidelity models. Another situation is when the "black-box" function is slightly altered, for example, a constant is changed due to the change of operating condition. Can we have a mechanism to fine tune the existing metamodel to adapt to such a change?

\section{Intelligent Sampling}

Current sampling schemes for metamodeling focus on the initial sampling in order to achieve certain space filling properties. As a matter of fact, if the function to be approximated is considered as "black-box," the adequate number of initial sample points will remain to be a mystery. Without knowing the adequate number of sample points, the distribution of the sample points becomes less important. Therefore, the subtle differences between various space filling sampling methods may not deserve so much attention. The focus on sampling, in the authors' opinion, should shift to how to "intelligently" generate minimum number of sample points so that the metamodel reflects the real "black-box" function in interested areas. This statement implies that the sampling process is iterative, and ought to be, progressive. Though there are methods on iterative sampling as reviewed before, more "intelligent" sampling schemes, which may be assisted by domain 
knowledge, need to be developed to further advance the metamodeling techniques.

\section{Summary}

This work provides an overview of the metamodeling techniques and their application to support product design. Research and development in metamodeling are categorized according to the needs of design engineers, i.e., approximation of computation-intensive processes, enhancement of the understanding of the design problem, assistance in defining the design optimization model, and support of various optimization needs. Challenges and future development are also discussed. It is hoped that this work can help researchers and engineers who just start in this area. Also it is hoped that this work will help current researchers and developers by being a reference and inspiration for future work.

\section{Acknowledgement}

Financial support from Natural Science and Engineering Research Council (NSERC) of Canada is appreciated.

\section{Bibliography}

Alexandrov, N., Dennis, J. E. J., Lewis, R. M., and Torczon, V. (1998). "A trust region framework for managing the use of approximation models in optimization." Structural Optimization, 15(1), 16-23.

Au, S. K., and Beck, J. L. (1999). "A new adaptive importance sampling scheme for reliability calculations." Structural Safety, 21, 135-158.

Bakr, M. H., Bandler, J. W., Madsen, K., and Sondergaard, J. (2000). "Review of the Space Mapping Approach to Engineering Optimization and Modeling." Journal of Optimization and Engineering, 1, 241-276.

Balabanov, V. O., Giunta, A. A., Golovidov, O., Grossman, B., Mason, W. H., and Watson, L. T. (1999). "Reasonable design space approach to response surface approximation." Journal of Aircraft, 36(1), 308-315.

Barthelemy, J. F. M., and Haftka, R. (1993). "Approximation Concepts for Optimal Structural Design -- A Review." Structural Optimization, 5, 129-144.

Batill, S. M., Stelmack, M. A., and Sellar, R. S. (1999). "Framework for Multidisciplinary Design Based on Response-Surface Approximations." Journal of Aircraft, 36(1), 287-297.

Booker, A. J., Dennis, J. E., Jr., F., P. D., Serafini, D. B., Torczon, V., and Trosset, M. W. (1999). "A Rigorous Framework for Optimization of Expensive Functions by Surrogates." Structural Optimization, 17(1), 1-13.

Booker, A. J., Meckesheimer, M., and Torng, T. (2004). "Reliability Based Design Optimization Using Design Explorer." Journal of Optimization and Engineering, 5, 179205.

Box, G. E. P., and Draper, N. R. (1969). Evolutionary Operation: A Statistical Method for Process Management, John Wiley \& Sons, Inc., New York.

Chen, W. (1995). "A Robust Concept Exploration Method for Configuring Complex System," Ph.D. Dissertation, Georgia Institute of Technology, Atlanta.

Chen, W., Allen, J. K., Schrage, D. P., and Mistree, F. (1997). "Statistical Experimentation Methods for Achieving
Affordable Concurrent Systems Design." AIAA Journal, 35(5), 893-900.

Chen, W., Allen, J. K., Tsui, K. L., and Mistree, F. (1996). "A Procedure for Robust Design: Minimizing Variations Caused by Noise Factors and Control Factors." Journal of Mechanical Design, Transactions of the ASME, 118, 478485.

Chen, W., Fu, W., Biggers, S. B., and Latour, R. A. (2000). "An Affordable Approach for Robust Design of Thick Laminated Composite Structure." Journal of Optimization and Engineering, 1(3), 305-322.

Clarke, S. M., Griebsch, J. H., and Simpson, T. W. (2005). "Analysis of Support Vector Regression for Approximation of Complex Engineering Analyses,." Transactions of ASME, Journal of Mechanical Design, in press.

Cresssie, N. (1988). "Spatial Prediction and Ordinary Kriging." Mathematical Geology, 20(4), 405-421.

Dennis, J. E., and Torczon, V. (1996). "Managing Approximation Models in Optimization." Multidisciplinary Design Optimization: State of the Art, N. Alexandrov and M. Y. Hussaini, eds., Society for Industrial and Applied Mathematics, Philadelphia.

Dyn, N., Levin, D., and Rippa, S. (1986). "Numerical Procedures for Surface Fitting of Scattered Data by Radial Basis Functions." SIAM Journal of Scientific and Statistical Computing, 7(2), 639-659.

Eddy, J., and Lewis, K. E. (2002a). "Multidimensional Design Visualization in Multiobjective Optimization." 9th AIAA/USAF/NASA/ISSMO Symposium on Multidisciplinary Analysis and Optimization, Atlanta, GA, AIAA-2002-5621.

Eddy, J., and Lewis, K. E. (2002b). "Visualization of Multidimensional Design and Optimization Data Using Cloud Visualization." ASME 2002 Design Engineering Techinical Conference and Computers and Information in Engineering Conference, Montreal, Canada, DETC2002/DAC-34130.

Ellman, T., Keane, J., Schwabacher, M., and Yao, K. T. (1997). "Multi-level Modeling for Engineering Design Optimization." Artificial Intelligence for Engineering Design, Analysis, and Manufacturing, 11(5), 1-36.

Fang, K. T., Lin, D. K. J., Winker, P., and Zhang, Y. (2000). "Uniform Design: Theory and Application." Technometrics, 39(3), 237-248.

Friedman, J. H. (1991). "Multivariate Adaptive Regressive Splines." The Annals of Statistics, 19(1), 1-67.

Fu, J. C., and Wang, L. (2002). "A random-discretization based Monte Carlo sampling method and its applications." Methodology and Computing in Applied Probability, 4, 5-25.

Gelsey, A., Schwabacher, M., and Smith, D. (1998). "Using Modeling Knowledge to Guide Design Space Search." Artificial Intelligence, 100(1-0), 1-27.

Giunta, A. A., Balabanov, V., Haim, D., Grossman, B., Mason, W. H., Watson, L. T., and Haftka, R. T. (1997). "Multidisciplinary Optimization of a Supersonic Transport Using Design of Experiments theory and Response Surface Modeling." Aeronautical Journal, 101(1008), 347-356.

Giunta, A. A., and Watson, L. T. (1998). "A Comparison of Approximation Modeling Techniques: Polynomial Versus Interpolating Models." Proceedings of the 7th AIAA/USAF/NASA/ISSMO Symposium on Multidisciplinary Analysis \& Optimization, St. Louis, MO, 392-401, AIAA-984758.

Golovidov, O., Kodiyalam, S., Marineau, P., Wang, L., and Rohl, P. (1998). "Flexible Implementation of Approximation Concepts in an MDO Framework." 7th AIAA/USAF/NASA/ISSMO Symposium on Multidisciplinary 
Analysis and Optimization, St. Louis, Missouri, AIAA 984959.

Gu, L. (2001). "A Comparison of Polynomial Based Regression Models in Vehicle Safety Analysis." 2001 ASME Design Engineering Technical Conferences - Design Automation Conference, Pittsburgh, PA, DAC-21063.

Hacker, K., Eddy, J., and Lewis, K. E. (2001). "Tuning a Hybrid Optimization Algorithm by Determining the Modality of the Design Space." ASME 2001 Design Engineering Technical Conferences and Computers and Information in Engineering Conference, Pittsburgh, PA, DETC2001/DAC-21093.

Haftka, R. T., Scott, E. P., and Cruz, J. R. (1998). "Optimization and Experiments: A Survey." Applied Mechanics Review, 51(7), 435-448.

Hedayat, A. S., Sloane, N. J. A., and Stufken, J. (1999). Orthogonal Arrays: Theory and Applications, Springer, New York.

Hirokawa, N., Fujita, K., and Iwase, T. (2002). "Voronoi Diagram Based Blending of Quadratic Response Surfaces for Cumulative Global Optimization." 9th AIAA/ISSMO Symposium on Multi-Disciplinary Analysis and Optimization, Atlanta, GA, AIAA-2002-5460.

Huber, K. P., Berthold, M. R., and Szczerbicka, H. (1996). "Analysis of Simulation Models with Fuzzy Graph Based Metamodeling." Performance Evaluation, 27-28, 473-490.

Iman, R. L., and Conover, W. J. (1980). "Small Sensitivity Analysis Techniques for Computer Models with an Application to Risk Assessment." Communication Statistics Theory and Methods, A9(17), 1749-1842.

Jin, R., Chen, W., and Simpson, T. W. (2001). "Comparative Studies of Metamodeling Techniques Under Multiple Modeling Criteria." Structural and Multidisciplinary Optimization, 23(1), 1-13.

Jin, R., Du, X., and Chen, W. (2003). "The Use of Metamodeling Techniques for Optimization Under Uncertainty." Structrual and Multidisciplinary Optimization, 25(2), 99-116.

Johnson, M. E., Moore, L. M., and Ylvisaker, D. (1990). "Minimax and Maximin Distance Designs." Journal of Statistical Planning and Inferences, 26(2), 131-148.

Jones, D. R., Schonlau, M., and Welch, W. J. (1998). "Efficient Global Optimization of Expensive Black Box Functions." Journal of Global Optimization, 13, 455-492.

Kalagnanam, J. R., and Diwekar, U. M. (1997). "An Efficient Sampling Technique for Off-Line Quality Control." Technometrics, 39(3), 308-319.

Keim, D. A., and Kriegel, H. P. (1996). "Visualization Techniques for Mining Large Databases: A Comparison." IEEE Transactions on Knowledge and Data Engineering, 8(6), 923-938.

Kleijnen, J. P. C., and van Beers, W. (2003). "Kriging for Interpolation in Random Simulation." Journal of the Operational Research Society, 54, 255-262.

Kloess, A., Mourelatos, Z., and Meernik, P. (2003). "Prababilistic Analysis of An Automotive Body-Door System." International Journal of Vehicle Design, 0(0), 1-26.

Koch, P. N., Simpson, T. W., Allen, J. K., and Mistree, F. (1999). "Statistical Approximations for Multidisciplinary Desgin Optimization: The Problem of Size." Journal of Aircraft, 36(1), 275-286.

Koehler, J. R. (1997). "Estimating the Response, Derivatives, and Transmitted Variance Using Computer Experiments." 1997 Symposium on the Interface of Computing Science and Statistics, Houston, TX
Langley, P., and Simon, H. A. (1995). "Applications of Machine Learning and Rule Induction." Communications of the ACM, 38(11), 55-64.

Leary, S. J., Bhaskar, A., and Keane, A. J. (2003). "A Knowledge-based Approach To Response Surface Modelling in Multifidelity Optimization." Journal of Global Optimization, 26, 297-319.

Li, Y., Fadel, G. M., and Wiecek, M. M. (1998). "Approximating Pareto curves using the hyper-ellipse." 7th AIAA/USAF/NASA/ISSMO Symposium on Multidisciplinary Analysis and Optimization, St. Louis, AIAA-98-4961.

Lophaven, S. N., Nielsen, H. B., and Søndergaard, J. (2002). "DACE - A Matlab Kriging Toolbox - Version 2.0." Report IMM-REP-2002-12, Informatics and Mathematical Modelling, Technical University of Denmark, Kgs. Lyngby, Denmark.

Madu, C. N. (1995). "A Fuzzy Theoretic Approach to Simulation Metamodeling." Appl. Math. Lett., 8(6), 35-41.

Martin, J. D., and Simpson, T. W. (2005). "Use of Kriging Models to Approximate Deterministic Computer Models." AIAA Journal, 43(4), 853-863.

Mattson, C. A., and Messac, A. (2002). "A Concept Selection in n-Dimension Using s-Pareto Frontiers and Visualization." 9th AIAA/USAF/NASA/ISSMO Symposium on Multidisciplinary Analysis and Optimization, Atlanta, GA, AIAA-2002-5418.

McKay, M. D., Bechman, R. J., and Conover, W. J. (1979). "A Comparison of Three Methods for Selecting Values of Input Variables in the Analysis of Output from a Computer Code." Technometrics, 21(2), 239-245.

Meckesheimer, M., Booker, A. J., Barton, R. R., and Simpson, T. W. (2002). "Computationally Inexpensive Metamodel Assessment Strategies." AIAA Journal, 40(10), 2053-2060.

Mitchell, T. J. (1974). "An Algorithm for the Construction of "D-Optimal" Experimental Designs." Technometrics, 16(2), 203-210.

Montgomery, D. (1991). Design and Analysis of Experiments, John Wiley and Sons, New York.

Morris, M. D., Mitchell, T. J., and Ylvisaker, D. (1993). "Bayesian Design and Analysis of Computer Experiments: Use of Derivatives in Surface Prediction." Technometrics, 35(3), 243-255.

Mullur, A. A., and Messac, A. (2005). "Extended Radial Basis Functions: More Flexible and Effective Metamodeling." AIAA Journal, accepted.

Myers, R. H., and Montgomery, D. (1995). Response Surface Methodology: Process and Product Optimization Using Designed Experiments, John Wiley and Sons, Inc., Toronto.

Oberkampf, W. L., and Trucano, T. G. (2000). "Validation Methodology in Computational Fluid Dynamics." Fluids 2000, Denver, CO, AIAA 2000-2549.

Ong, Y. S., Nair, P. B., and Keane, A. J. (2003). "Evolutionary Optimization of Computationally Expensive Problems via Surrogate Modeling." AIAA Journal, 41(4), 687-696.

Osio, I. G., and Amon, C. H. (1996). "An Engineering Design Methodology with Multistage Bayesian Surrogates and Optimal Sampling." Research in Engineering Design, 8(4), 189-206.

Owen, A. (1992). "Orthogonal Arrays for Computer Experiments, Integration, and Visualization." Statistica Sinica(2), 439-452.

Papadrakakis, M., Lagaros, M., and Tsompanakis, Y. (1998). "Structrual Optimization Using Evolution Strategies and Neural Networks." Computer Methods in Applied Mechanics and Engineering, 156(1-4), 309-333. 
Park, J. S. (1994). "Optimal Latin-hypercube Designs for Computer Experiments." Journal of Statistical Planning Inference, 39, 95-111.

Pérez, V. M., Renaud, J. E., and Watson, L. T. (2002). "Adaptive Experimental Design for Construction of Response Surface Approximations." AIAA Journal, 40(12), 2495-2503.

Rasmussen, J. (1998). "Nonlinear Programming by Cumulative Approximation Refinement." Structural Optimization, 15, 17.

Renaud, J. E., and Gabriele, G. A. (1994). "Approximation in Non-hierarchical System Optimization." AIAA Journal, 32, 198-205.

Roache, P. J. (1998). Verification and Validation in Computational Science and Engineering, Hermosa Publishers.

Rodríguez, J. F., Pérez, V. M., Padmanabhan, D., and Renaud, J. E. (2001). "Sequential Approximate Optimization Using Variable Fidelity Response Surface Approximations." Structural and Multidisciplinary Optimization, 22, 24-44.

Rodríguez, J. F., Renaud, J. E., and Watson, L. T. (1998). "Trust Region Augmented Lagrangian Methods for Sequential Response Surface Approximation and Optimization." Transactions of ASME, Journal of Mechanical Design, 120, 58-66.

Sacks, J., B., S. S., and Welch, W. J. (1989a). "Designs for Computer Experiments." Technometrics, 31(1), 41-47.

Sacks, J., Welch, W. J., Mitchell, T. J., and Wynn, H. P. (1989b). "Design and Analysis of Computer Experiments." Statistical Science, 4(4), 409-435.

Sahin, K. H., and Diwekar, U. M. (2004). "Better Optimization of Nonlinear Uncertain Systems (Bonus): A New Algorithm for Stochastic Programming Using Reweighting Through Kernel Density Estimation." Annals of Operation Research, 132, 47-68.

Sasena, M., Papalambros, P., and Goovaerts, P. (2002). "Global Optimization of Problems with Disconnected Feasible Regions Via Surrogate Modeling." 9th AIAA/ISSMO Symposium on Multidisciplinary Analysis and Optimization, Atlanta, Georgia, AIAA 2002-5573.

Schonlau, M. S., Welch, W. J., and Jones, D. R. (1998). "Global Versus Local Search in Constrained Optimization of Computer Models." New Development and Applications in Experimental Design, N. Flournoy, W. F. Rosenberger, and W. K. Wong, eds., Institute of Mathematical Statistics, 1125.

Shan, S., and Wang, G. G. (2003). "Introducing rough set for design space exploration and optimization." ASME 2003 Design Engineering Technical Conferences and Computers and Information in Engineering Conference, Chicago, Illinois, USA, DAC48761.

Shan, S., and Wang, G. G. (2004). "An Efficient Pareto Set Identification Approach for Multi-objective Optimization on Black-box Functions." Transactions of the ASME, Journal of Mechanical Design, In press.

Shin, Y. S., and Grandhi, R. V. (2001). "A Global Structural Optimization Technique Using an Interval Method." Structural and Multidisciplinary Optimization, 22, 351-363.

Simpson, T. W. (2004). "Multidisciplinary Design Optimization." Aerospace America, 34.

Simpson, T. W., Booker, A. J., Ghosh, D., Giunta, A. A., Koch, P. N., and Yang, R. J. (2004). "Approximation methods in multidisciplinary analysis and optimization: a panel discussion." Structural and Multidisciplinary Optimization, 27, 302-313.
Simpson, T. W., Lin, D. K. J., and Chen, W. (2001a). "Sampling Strategies for Computer Experiments: Design and Analysis." International Journal of Reliability and Application, 2(3), 209-240.

Simpson, T. W., Mauery, T. M., Korte, J. J., and Mistree, F. (2001b). "Kriging Metamodels for Global Approximation in Simulation-based Multidisciplinary Design Optimization." AIAA Journal, 39(12), 2233-2241.

Simpson, T. W., Peplinski, J., Koch, P. N., and Allen, J. K. (2001c). "Metamodels for Computer-Based Engineering Design: Survey and Recommendations." Engineering with Computers, 17(2), 129-150.

Sobieski, I., and Kroo, I. (2000). "Collaborative Optimization Using Response Surface Estimation." AIAA Journal, 38(10), 1931-1938.

Sobieszczanski-Sobieski, J., and Haftka, R. T. (1997). "Multidisciplinary Aerospace Design Optimization: Survey of Recent Developments." Structural Optimization, 14(1), 123.

Srivastava, A., Hacker, K., Lewis, K. E., and Simpson, T. W. (2004). "A Method for Using Legacy Data for Metamodelbased Design of Large-Scale Systems." Structrual and Multidisciplinary Optimization, 28, 146-155.

Stump, G., Simpson, T. W., Yukish, M., and Bennett, L. (2002). "Multidimensional Design and Visualization and Its Application to a Design By Shopping Paradigm." 9th AIAA/USAF/NASA/ISSMO Symposium on Multidisciplinary Analysis and Optimization, Atlanta, GA, AIAA-2002-5622.

Taguchi, G., Yokoyama, Y., and Wu, Y. (1993). Taguchi Methods: Design of Experiments, American Supplier Institute, Allen Park, Michigan.

Tang, B. (1993). "Orthogonal Array-based Latin Hypercubes." Journal of American Statistical Association, 88(424), 13921397.

Tappeta, R. V., and Rosenberger, W. F. (2001). "Interactive multiobjective optimization design strategy for decision based design." Journal of Mechanical Design, Transactions of the ASME, 123, 205-215.

Toropov, V., van Keulen, F., Markine, V., and de Doer, H. (1996). "Refinements in the Multi-Point Approximation Method to Reduce the Effects of Noisy Structural Responses." 6th AIAA/USAF/NASA/ISSMO Symposium on Multidisciplinary Analysis and Optimization, Bellevue, WA, 941-951

Toropov, V. V., and Filatov, A. A. (1993). "Multi-parameter Structrual Optimization Using FEM and Multipoint Approximation." Structural and Multidisciplinary Optimization, 6, 7-14.

Turner, C. J., and Crawford, R. H. (2005). "Selecting an Appropriate Metamodel: The Case for NURBS Meamodels." ASME 2005 Design Engineering Technical Conferences and Computers and Information in Engineering Conference, Long Beach, California, DETC2005-85043.

Varadarajan, S., Chen, W., and Pelka, C. J. (2000). "Robust Concept Exploration of Propulsion Systems with Enhanced Model Approximation Capabilities." Engineering Optimization, 32(3), 309-334.

Wang, D. (2005). "Multidisciplinary Design Optimization with Collaboration Pursuing and Domain Decomposition: Application to Aircraft Design," Ph.D. Dissertation, University of Manitoba, Winnipeg.

Wang, G. G. (2003). "Adaptive Response Surface Method Using Inherited Latin Hypercube Design Points." Transactions of ASME, Journal of Mechanical Design, 125, 210-220. 
Wang, G. G., Dong, Z., and Aitchison, P. (2001). "Adaptive Response Surface Method - A Global Optimization Scheme for Computation-intensive Design Problems." Journal of Engineering Optimization, 33(6), 707-734.

Wang, G. G., and Simpson, T. W. (2004). "Fuzzy Clustering Based Hierarchical Metamodeling for Space Reduction and Design Optimization." Journal of Engineering Optimization, 36(3), 313-335.

Wang, G. G., Wang, L., and Shan, S. (2005). "Reliability Assessment Using Discriminative Sampling and Metamodeling." 2005 SAE World Congress, Cobo Center, Detroit, MI, USA, 05M-400

Wang, L., Shan, S., and Wang, G. G. (2004). "Mode-Pursuing Sampling Method for Global Optimization on Expensive Black-box Functions." Journal of Engineering Optimization, 36(4), 419-438.

Wang, L. P., Grandhi, R. V., and Canfield, R. A. (1996). "Multivariate Hermite Approximation for Design Optimization." International Journal for Numerical Methods in Engineering, 39, 787-803.

Welch, W. J., Buck, R. J., Sacks, J., Wynn, H. P., Mitchell, T. J., and Morris, M. D. (1992). "Screening, Predicting, and Computer Experiments." Technometrics, 34(1), 15-25.

Wilson, B., Cappelleri, D. J., Simpson, T. W., and Frecker, M. I. (2000). "Efficient Pareto frontier exploration using surrogate approximations." 8th AIAA/USAF/NASA/ISSMO Symposium on Multidisciplinary Analysis and Optimization, Long Beach, CA, AIAA-2000-4895.

Winer, E. H., and Bloebaum, C. L. (2002a). "Development of visual design steering as an aid in large-scale multidisciplinary design optimization. Part I: method development." Structural and Multidisciplinary Optimization, 23(6), 412 - 424.

Winer, E. H., and Bloebaum, C. L. (2002b). "Development of visual design steering as an aid in large-scale multidisciplinary design optimization. Part II: method validation." Structural and Multidisciplinary Optimization, 23(6), 425 - 435.

Wong, P. C., and Bergeron, R. D. (1997). "30 Years of Multidimensional Multivariate Visualization." Scientific Visualization - Overviews, Methodologies and Techniques, G. M. Nielson, H. Hagan, and H. Muller, eds., IEEE Computer Society Press, Los Alamitos, CA, 3-33.

Wujek, B. A., and Renaud, J. E. (1998a). "New adaptive movelimit management strategy for approximate optimization, Part 1." AIAA Journal, 36(10), 1911-1921.

Wujek, B. A., and Renaud, J. E. (1998b). "New adaptive movelimit management strategy for approximate optimization, Part 2." AIAA Journal, 36(10), 1922-1934.

Yang, B. S., Yeun, Y. S., and Ruy, W. S. (2003). "Managing Approximation Models in Multiobjective Optimization." Structrual and Multidisciplinary Optimization, 24, 141-156.

Ye, K. Q., Li, W., and Sudianto, A. (2000). "Algorithmic Construction of Optimal Symmetric Latin Hypercube Designs." Journal of Statistical Planning and Inferences, 90, 145-159.

Youn, B. D., and Choi, K. K. (2004). "Selecting Probabilistic Approaches for Reliability-Based Design Optimization." AIAA Journal, 42(1), 124-131.

Zhang, J., Wiecek, M. M., and Chen, W. (2000). "Local Approximation of the Efficient Frontier in Robust Design." Transactions of ASME, Journal of Mechanical Design, 122(232-236).

Zou, T., Mahadevan, S., Mourelatos, Z., and Meernik, P. (2002). "Reliability Analysis of Automotive Body-door Subsystem." Reliability Engineering and System Safety, 78, 315-324.

Zou, T., Mourelatos, Z., Mahadevan, S., and Tu, J. (2003). "An Indicator Response Surface-Based Monte Carlo Method for Efficient Component and System Reliability Analysis." ASME 2003 Design Engineering Technical Conferences and Computers and Information in Engineering Conference, Chicago, Illinois USA, DAC-48708. 